$\mathrm{AP} B=$

\title{
Florística e fitossociologia de áreas de restauração com diferentes históricos de uso
}

\author{
Francisca Alcivania Melo Silva ${ }^{*}$ (D) Gilvani Scatolin Leite ${ }^{2}$ (D) Ocimar José Baptista Bim ${ }^{3}$ (D) \\ ${ }^{1}$ Universidade Estadual Paulista Julio de Mesquita Filho, Câmpus de Registro, Rua Nelson Brihi Badur, 430, CEP 11900-000, Registro, SP, Brasil \\ ${ }^{2}$ Universidade Federal da Fronteira Sul, Rodovia BR-158, s/n - Zona Rural, CEP 85301-970, Laranjeira do Sul, PR, Brasil \\ IInstituto Florestal, Avenida Clara Gianotti de Souza, 1139, CEP 11900-000, Registro, SP, Brasil
}

\section{"Autor correspondente: \\ alcivania.silva@unesp.br}

Termos para indexação:

Floresta Ombrófila Densa Submontana

Restauração de Terras

Sucessão Secundária

Index terms:

Submontane Dense Ombrophylous Forest Land restoration

Secondary sucession

Histórico do artigo:

Recebido em 15/03/2019

Aprovado em 26/11/2020

Publicado em 31/05/2021

\begin{abstract}
Resumo - Foi realizado um estudo florístico e fitossociológico em áreas no Parque

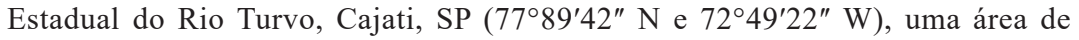
restauração com três anos em bananal abandonado (Rest 3) e outra área de restauração com cinco anos em pastagem degradada (Rest 5). Em cada área foram instaladas seis parcelas de $100 \mathrm{~m}^{2}$, analisando-se diâmetro e altura de todos os indivíduos com altura superior a $50 \mathrm{~cm}$ e fazendo-se amostragem de solos para fertilidade na profundidade $0-20 \mathrm{~cm}$. A estrutura da comunidade foi descrita a partir dos estimadores fitossociológicos: frequência relativa, dominância relativa e valor de importância. A diversidade foi calculada pelo índice de Shannon e equabilidade pelo índice de Pielou. Na Rest 5 foram registrados 2.117 ind ha ${ }^{-1}$ (18 famílias e 39 espécies) e na Rest 3 foram 4.650 ind ha ${ }^{-1}$ ( 21 famílias e 54 espécies). Os parâmetros fitossociológicos refletem áreas em processos iniciais de sucessão secundária, caracterizando-se o uso anterior como bananal em um ambiente mais favorável para o desenvolvimento das espécies plantadas e estabelecimento de propágulos.
\end{abstract}

\section{Floristics and phytosociology of restoration areas with different history of use}

\begin{abstract}
The dynamics of floristic and fitossociology composition in the restoration process were studied on areas within the Rio Turvo State Park, in Cajati, State of São Paulo $\left(77^{\circ} 89^{\prime} 42^{\prime \prime} \mathrm{N}\right.$ and $\left.72^{\circ} 49^{\prime} 22^{\prime \prime} \mathrm{W}\right)$, in a restoration area over three years, on an abandoned banana plantation (Rest 3 ) and in a restoration area over five years, on a degraded area of grazing land (Rest 5). In each area, six plots were installed (100 $\mathrm{m}^{2}$ each). We analysed the diameter and height of all individuals over $50 \mathrm{~cm}$ of height and we collected soil samples for fertility analysis ( $0-20 \mathrm{~cm}$ depth). The structure of the community was described based on phytosociological estimators (relative density, relative dominance, importance value). Diversity was calculated using Shannon's diversity index, and evenness by Pielou's index. There were 2,117 ind ha- ${ }^{-1}(18$ families and 39 species) in Rest 5 and 4,650 ind ha ${ }^{-1}$ (21 families and 54 species) in Rest 3 . The phytosociological parameters reflect areas in the initial stages of secondary succession, where previous use as a banana plantation incurred in the composition of a more favorable environment for the development of planted species and establishment of propagules.
\end{abstract}




\section{Introdução}

A restauração ecológica é o processo de auxiliar a recuperação de um ecossistema que foi degradado, danificado ou destruído (Society for Ecological Restoration, 2004). Numa visão mais abrangente e atual, a restauração ecológica considera não só aspectos ecológicos, que tratam do restabelecimento da biodiversidade e dos processos ecológicos nos ecossistemas, mas também aspectos econômicos e sociais relacionados à restauração. Durante o processo dinâmico de recomposição florística, haverá inúmeros processos ecológicos ocorrendo, como crescimento dos indivíduos arbóreos, aumento da micro e macrofauna do solo, descompactação do solo com a ação dos micro-organismos e raízes, diminuição de espécies invasoras, regeneração natural, mortalidade das espécies, intempéries climáticas, entre outros (Brancalion et al., 2009).

As técnicas de restauração de ecossistemas degradados incluem a eliminação da fonte de distúrbios, intervenções no solo, eliminação ou manejo de espécies invasoras e introdução de espécies. Em restaurações florestais, a introdução de espécies é geralmente feita por plantio de mudas. Um dos objetivos dessa etapa é atrair dispersores e estabelecer espécies lenhosas a partir da melhoria das condições de sombreamento e temperatura nessas áreas (Society for Ecological Restoration, 2004; Holl, 2013; Fonseca et al., 2017).

O Vale do Ribeira, São Paulo, região que abriga a maior extensão contínua e conservada da Mata Atlântica no Brasil, foi considerado como de extrema importância biológica para a conservação da biodiversidade da Mata Atlântica (Brasil, 2000). A Bacia Hidrográfica do Ribeira de Iguape, no Litoral Sul, concentra 1.163.515 milhões de ha de vegetação natural remanescente, que corresponde a 53,4\% de sua superfície, representando $4,7 \%$ da vegetação natural do estado de São Paulo, dos quais 35,6\% (414.002 ha) estão inseridos em unidades de conservação (UCs) (Kronka et al., 2007; Nalon et al., 2008; Xavier et al., 2008). Nessas UCs, um dos desafios é restaurar áreas degradadas antes da incorporação pelos principais usos do solo na região (bananicultura e pastagem).

De acordo com Chazdon (2008), existem poucos estudos sobre restauração ecológica com diferentes tratamentos (incluindo regeneração natural), que considerem o histórico de uso da área, os solos, a proximidade com as fontes de sementes e o tempo de abandono da área. Moura \& Mantovani (2017), descrevendo a evolução do processo de restauração da vegetação em áreas antes ocupadas pela bananicultura, observaram que esses ambientes se mostraram capazes de desencadear os processos de regeneração natural com elevada riqueza de espécies e densidade de indivíduos, apenas com a interrupção dos tratos culturais. Tal comportamento foi atribuido ao histórico de uso e à presença de remanescentes florestais (fonte de propágulos) próximos à área. A condução da regeneração natural e o plantio de mudas, se realizados próximos a fragmentos florestais, favorecem a ação de dispersores, acelerando o processo de sucessão, em virtude da formação de uma comunidade mais abundante e biodiversa (Suganuma et al., 2014, 2018; Holl et al., 2017).

Mônico (2019) testou, em pastagens vizinhas a fragmentos de Mata Atlântica, diferentes estratégias de restauração na estruturação de comunidades florestais tropicais, quanto à eficiência ecológica e custos. Este autor concluiu que quanto mais intensivos os manejos adotados (fertilização e controle de invasoras), maior a previsibilidade das comunidades formadas e o sucesso da restauração.

Esse estudo objetivou comparar dois fragmentos de floresta em processo inicial de restauração, pela descrição da florística e da fitossociologia, a fim de responder as seguintes questões: Como diferentes históricos de uso e fertilidade do solo influenciam a composição e fitossociologia da comunidade?; A regeneração da comunidade arbórea nas restaurações é afetada pelo histórico de uso?

\section{Material e métodos}

O estudo foi realizado no Núcleo Capelinha do Parque Estadual do Rio Turvo (PERT), localizado entre os municípios de Cajati e Barra do Turvo, sul do Estado de São Paulo (Figura 1) (c7789'42" N e 7249'22" W). O PERT pertence ao Mosaico do Jacupiranga (MOJAC) e possui uma área de aproximadamente $2.439 \mathrm{~km}^{2}$. A área é coberta, principalmente, por Floresta Ombrófila Densa Submontana (IBGE, 2012). De acordo com a classificação climática de Köppen (Köppen \& Geiger, 1928), o clima regional é do tipo Cfa - Cfb, com temperatura média do mês mais quente superior a $18^{\circ} \mathrm{C}$. 
A temperatura do mês mais quente varia entre $24{ }^{\circ} \mathrm{C}$ e $25^{\circ} \mathrm{C}$, correspondendo aos meses de janeiro e fevereiro. A precipitação média anual da região do Vale do Ribeira está em torno de $1.500 \mathrm{~mm}$, com uma variação positiva ou negativa em torno de $250 \mathrm{~mm}$ (Alvares et al., 2013). A média total de chuvas durante o mês mais frio alcança $60 \mathrm{~mm}$. O solo da área foi classificado como Cambissolo Háplico distrófico (São Paulo, 2017).

Foram levantadas informações com gestores do PERT, sendo prospectadas duas áreas em processo de restauração com diferentes históricos de uso (pastagem e bananal abandonado). As duas áreas amostradas encontram-se a $700 \mathrm{~m}$ de remanescentes florestais de Floresta Ombrófila Densa Submontana pertencentes ao PERT. As ações para restauração das áreas foram propostas pela Equipe de gestão do Parque na fase de prospecção das áreas do estudo (Ocimar Bim, comunicação pessoal ${ }^{1}$ ).

A área de pastagem (Rest 5) apresentava 2,23 ha. Estava em processo de restauração há cinco anos: na década de 1960 esta área era utilizada para produção de banana. A partir da década de 1970 o cultivo de banana foi substituído pela implantação de pastagem para a criação de bovinos. A pastagem não era manejada, e era formada predominantemente pela gramínea Urochloa decumbens (Stapf) R.D. Webster, sem qualquer aplicação de insumos. Esta situação perdurou até 2009, quando a área foi desapropriada por estar inserida nos limites do PERT (Ocimar Bim, comunicação pessoal ${ }^{1}$ ). Em 2010, foi iniciado o processo de restauração com o plantio de espécies nativas em espaçamento de 3,0 $\mathrm{m}$ x 2,0 m (1.666 ind ha ${ }^{-1}$ ), de acordo com o método proposto pelo Pacto pela Restauração da Mata Atlântica (Rodrigues et al., 2011). Não há informações sobre a proporção de indivíduos por espécie inicialmente plantados na área. A distribuição das espécies no momento do plantio ocorreu de forma sistemática, sendo alternadas nas linhas espécies pioneiras e não pioneiras A manutenção foi feita por meio de roçadas e coroamento das mudas, iniciando-se aos 2 meses após o plantio e mantendo-se uma frequência de 3 vezes por ano, nos 3 primeiros anos.
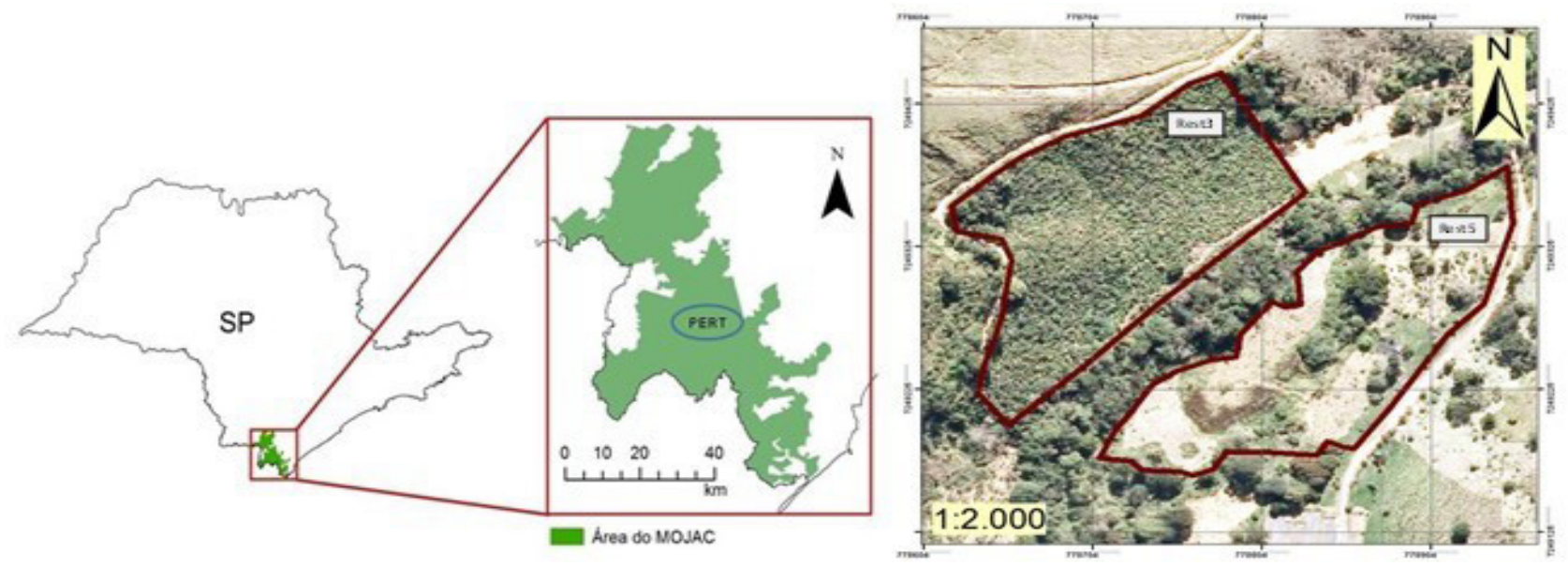

Figura 1. Localização das áreas de estudo no Parque Estadual do Rio Turvo, SP.

Figure 1. Location of the study areas in the Rio Turvo State Park, SP, Brazil.

A área de bananal abandonado (Rest 3) apresentava 2,50 ha. Estava há três anos em processo de restauração: na década de 1990, a área foi desmatada, com supressão de vegetação em estágio médio de regeneração para implantação de roças com diversas culturas. Em 2000 foi implantada a cultura da banana, com aplicação de $300 \mathrm{~g}$ de NPK e $200 \mathrm{~g}$ de calcário por planta de duas a três vezes ao ano. O bananal perdurou até 2012, quando houve a ação de reintegração de posse e incorporação ao PERT, semelhante ao que ocorreu na Rest 5. A área foi destinada à implantação do projeto de restauração florestal nas mesmas condições da Rest 5 (Ocimar Bim, comunicação pessoal ${ }^{1}$ ).

As áreas de estudo foram subdivididas em parcelas de $100 \mathrm{~m}^{2}$ (4 m x $25 \mathrm{~m}$ ), utilizando-se o programa de geoprocessamento ArcGIS 10.2. As linhas das 
quadrículas foram rotuladas com letras (A a I) e as colunas com números naturais inteiros ( 1 a 6 ), procedendo-se assim o sorteio. Em cada área foram sorteadas seis parcelas, número recomendado pelo Protocolo de monitoramento para programas e projetos de restauração florestal(Rodrigues et al., 2013).

Procedeu-se então a amostragem de solos em cada parcela (5 amostras simples, formando uma composta), à profundidade de $0-20 \mathrm{~cm}$. As amostras foram enviadas para o laboratório de Fertilidade do Solo da UNESP de Botucatu, SP, sendo determinados $\mathrm{pH}$, complexo sortivo e saturação por bases, de acordo com metodologia da Embrapa (Classen, 1997).

Em cada parcela, foram amostrados todos os indivíduos arbóreos plantados e também os regenerantes com altura $\mathrm{H} \geq 50 \mathrm{~cm}$. Cada indivíduo teve sua $\mathrm{H}$ medida com régua ou vara graduada, desde o nível do solo até o último par de folhas. Todos os indivíduos com altura $\geq 1,50 \mathrm{~m}$, tiveram seu diâmetro a $1,30 \mathrm{~m}$ do solo (DAP) medido, com auxílio de suta ou paquímetro. A identificação das espécies em campo se deu com apoio de um técnico do PERT; as demais foram coletadas para posterior identificação no Instituto Florestal. A identificação taxonômica seguiu o sistema proposto por APG IV (2016) para o grupo das Angiospermas. Os nomes científicos, bem como sua autoria, foram confirmados de acordo com Brazil Flora G (2021).

As espécies foram organizadas em listagens por famílias, grupos sucessionais (pioneiros e não pioneiros) e síndromes de dispersão (anemocóricas, zoocóricas e autocóricas), de acordo com Barbosa et al. (2017). Os valores de altura dos indivíduos regenerantes foram distribuídos nas seguintes classes: Classe I - 0,5 m $\leq \mathrm{h}$ $\leq 0,99 \mathrm{~m}$; Classe II $-1 \mathrm{~m} \leq \mathrm{h} \leq 2,99 \mathrm{~m}$; Classe III - 3,0 $\mathrm{m} \leq \mathrm{h} \leq 4,99 \mathrm{~m}$; Classe IV: $\mathrm{h} \geq 5,0 \mathrm{~m}$.

Com auxílio do programa Fitopac (Shepherd, 2010), realizaram-se os cálculos dos seguintes parâmetros fitossociológicos: densidade total por área, densidade específica relativa, frequência absoluta, frequência relativa, dominância por área, dominância relativa por espécie, valor de importância (Mueller-Dombois \& Ellenberg, 1974) e índice de diversidade de Shannon (Shannon, 1948). Utilizou-se o teste de Kruskal-Wallis para a análise das diferenças entre estimadores de área basal, altura média e densidade, além do percentual de indivíduos regenerantes e plantados nas áreas avaliadas.

\section{Resultados}

\section{Fertilidade do solo}

Os resultados das análises químicas dos solos das duas áreas avaliadas estão apresentados na Tabela 1.

\section{Levantamento Florístico e Fitossociológico}

$\mathrm{Na}$ Rest 5 foi registrado um total de 127 indivíduos (entre plantados e regenerantes), distribuídos em 18 famílias e 39 espécies, enquanto na área Rest 3 foram registrados 279 indivíduos, distribuídos em 21 famílias e 54 espécies.

Quando considerados os grupos ecológicos das espécies nas duas áreas, verificou-se predominância de não pioneiras, sendo isso mais evidente na Rest 3. A Rest 5 apresentou entre as espécies plantadas $25 \%$ de pioneiras e $75 \%$ de não pioneiras e a Rest 3, 30\% de pioneiras e $70 \%$ de não pioneiras.

A síndrome de dispersão zoocórica foi maior que a anemocórica e a autocórica nas duas áreas avaliadas, representado 55\% das espécies plantadas para a Rest 5 e $45 \%$ para a Rest 3. Entre as espécies regenerantes, a síndrome de dispersão zoocórica representou $67 \%$ na Rest 5 e $72 \%$ na Rest 3.

$\mathrm{Na}$ Rest 5, as famílias que apresentaram maior riqueza de espécies foram Fabaceae (12) e Myrtaceae (6), o mesmo ocorrendo na Rest 3 , com Fabaceae (15) e Myrtaceae (6).

Tabela 1. Caracterização química das amostras de solo $(0$ - $20 \mathrm{~cm})$ em áreas sob restauração no Parque Estadual do Rio Turvo, SP. Table 1. Chemical characterization of the soil samples $(0-20 \mathrm{~cm})$ in restorations areas s in the Rio Turvo State Park, SP, Brazil.

\begin{tabular}{|c|c|c|c|c|c|c|c|c|c|c|c|}
\hline & $\begin{array}{r}\mathrm{pH} \\
\mathrm{CaCl}_{2} \\
\end{array}$ & $\begin{array}{c}\mathrm{MO} \\
\left(\mathrm{g} \mathrm{dm}^{-3}\right)\end{array}$ & $\begin{array}{c}\mathrm{P} \\
\left(\mathrm{mg} \mathrm{dm}^{-3}\right)\end{array}$ & $\mathrm{Al}^{3+}$ & $\mathrm{H}+\mathrm{Al}$ & $\begin{array}{r}\mathrm{K} \\
\mathrm{mmol}\end{array}$ & $\mathrm{Ca}$ & $\mathrm{Mg}$ & S & CTC & $\begin{array}{l}\mathrm{V} \\
(\%)\end{array}$ \\
\hline Rest 5 & 5,1 & 23 & 6,0 & 1,0 & 33 & 1,7 & 30 & 10,0 & 41,7 & 74,7 & 56 \\
\hline Rest 3 & 5,7 & 22 & 16,0 & 0,0 & 19 & 1,8 & 63 & 33 & 100,8 & 119,8 & 84 \\
\hline
\end{tabular}

Rest 5 = área de pastagem em processo de restauração há cinco anos; Rest 3 = área de bananal abandonado há três anos em processo de restauração; MO = matéria orgânica; CTC = capacidade de troca de cátions; V= saturação por bases. 
Quanto ao número de indivíduos por família (Figura 2), destacaram-se na Rest 5 Fabaceae (40), Anacardiaceae (25) e Myrtaceae (20). Na Rest 3 destacaram-se as famílias Fabaceae (100), Myrtaceae (40) e Rutaceae (20).

Quando avaliado o número e percetual de espécies plantadas e regenerantes (Figura 3) nas duas áreas de restauração com diferentes idades, a Rest 3 apresentou 18 espécies plantadas e 36 regenerantes e na Rest 5 foram contabilizadas 19 espécies plantadas e 20 regenerantes.

Foram registradas nas 2 áreas avaliadas espécies regenerantes colonizadoras (não plantadas), sendo 7 espécies na Rest 5 (Tabela 2) e 26 na Rest 3 (Tabela 3). Essas espécies, em sua maioria, caracterizam-se pela síndrome de dispersão zoocórica.
$\mathrm{Na}$ Rest 5, do total de regenerantes, $61 \%$ pertencem ao grupo das pioneiras, enquanto enquanto $45 \%$ são pioneiras na Rest 3. A família Fabaceae se destacou na regeneração natural em número de indivíduos para as duas áreas avaliadas. Entre as espécies de regenerantes, apenas Alchornea glandulosa, Cecropia hololeuca, Ocotea tabacifolia, Senna multijuga, S. penducula, Solanum caavurana, S. paniculatum e Syagrus romanzoffiana foram comuns às duas áreas.

As nove espécies com valor de importância (VI) maior na Rest 5 representam 52\% do VI total (Tabela 2). Na Rest 3 , as dez espécies com maiores índices fitossociológicos representaram VI total $>50 \%$ (Tabela 3 ).

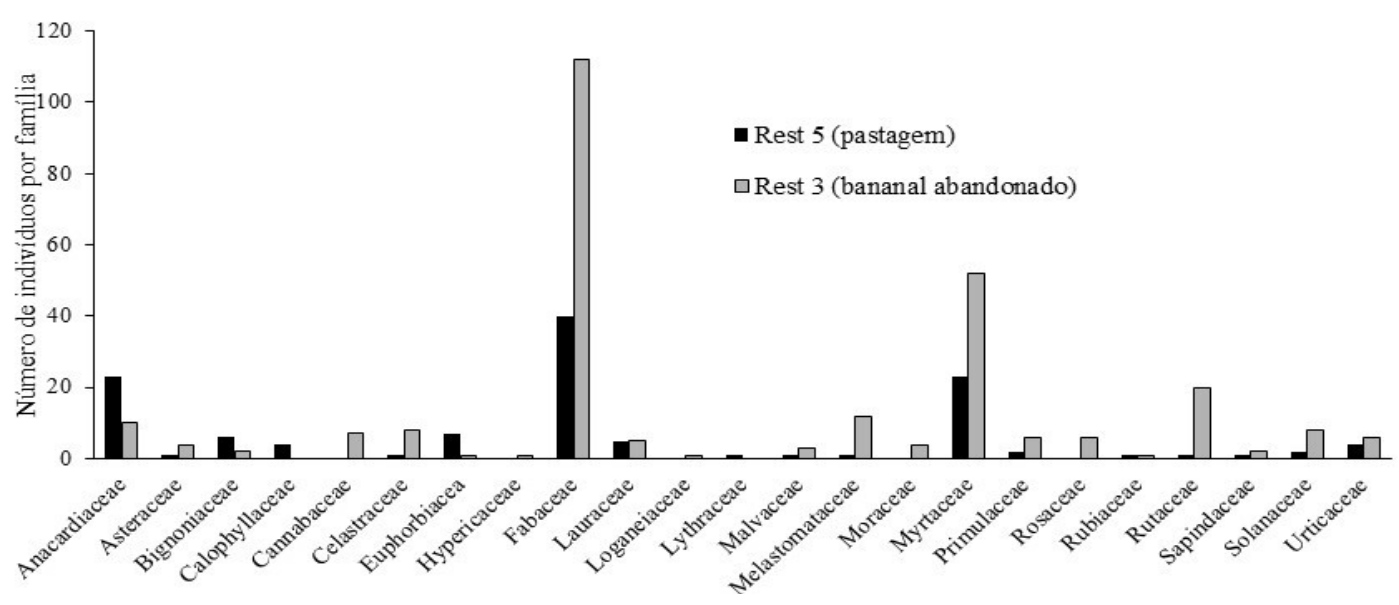

Figura 2. Número de indivíduos por família em áreas no Parque Estadual do Rio Turvo, SP. Rest $5=$ área de pastagem em processo de restauração há cinco anos; Rest 3 = área de bananal abandonado há três anos em processo de restauração.

Figure 2. Number of individuals per family in restoration areas in Rio Turvo State Park, São Paulo, Brazil. Rest $5=$ restoration area over five years, on a degraded area of grazing land; Rest 3 = restoration area over three years, on an abandoned banana plantation.

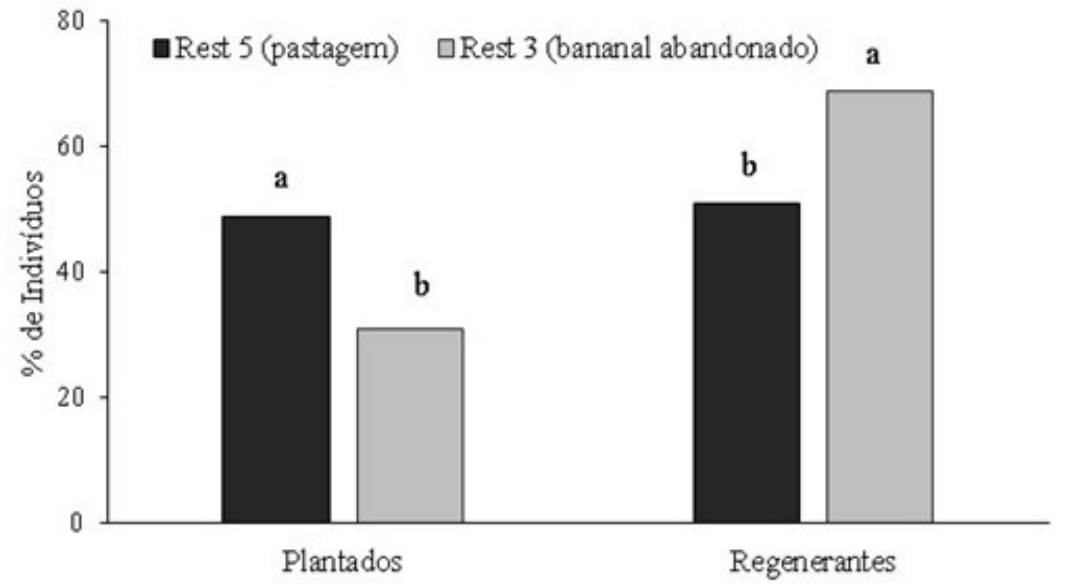

Figura 3. Percentual de espécies plantadas e de regenerantes em áreas de restauração no Parque Estadual do Rio Turvo, SP. Médias seguidas por letras iguais não diferem entre si pelo teste de Kruskall-Wallis $(\mathrm{p} \leq 0,05)$. Rest $5=$ área de pastagem em processo de restauração há cinco anos; Rest 3 = área de bananal abandonado há três anos em processo de restauração.

Figure 3. Percentage of regenerating and planted individuals in restoration areas in Rio Turvo State Park, SP, Brazil. Averages followed by the same letter, do not differ by the test of Kruskall-Wallis $(\mathrm{p} \leq 0.05)$. Rest $5=$ restoration area over five years, on a degraded area of grazing land; Rest $3=$ restoration area over three years, on an abandoned banana plantation. 
Tabela 2. Parâmetros fitossociológicos das espécies em área de restauração sobre pastagem após 5 anos (Rest 5) no Parque Estadual do Rio Turvo,SP.

Table 2. Phytosociological parameters of the species in restoration areas on pasture over 5 years (Rest 5) in the Rio Turvo State Park, SP, Brazil.

\begin{tabular}{|c|c|c|c|c|c|c|}
\hline ESPÉCIE & $\begin{array}{c}\text { NI } \\
\text { (PL) }\end{array}$ & $\begin{array}{c}\text { NI } \\
\text { (REG) }\end{array}$ & $\begin{array}{l}\text { DR } \\
\text { (\%) }\end{array}$ & $\begin{array}{c}\text { FR } \\
(\%)\end{array}$ & $\begin{array}{c}\text { DoR } \\
(\%)\end{array}$ & $\begin{array}{c}\text { VI } \\
(\%)\end{array}$ \\
\hline Syagrus romanzoffiana (Cham.) Glassman & 1 & 2 & 2,36 & 1,35 & 40,60 & 14,77 \\
\hline Lithrea molleoides (Vell.) Engl. & 15 & 8 & 18,11 & 4,05 & 4,27 & 8,81 \\
\hline Cecropia hololeuca Miq. & 0 & 4 & 3,15 & 2,70 & 16,67 & 7,51 \\
\hline Machaerium acutifolium Benth. & 5 & 13 & 14,17 & 1,35 & 0,85 & 5,46 \\
\hline Campomanesia guazumifolia (Cambess.) O. Berg & 5 & 3 & 6,30 & 5,41 & 0,43 & 4,04 \\
\hline Croton urucurana Baill. & 3 & 3 & 4,72 & 5,41 & 0,43 & 3,52 \\
\hline Eugenia uniflora L. & 4 & 2 & 4,72 & 5,41 & 0,43 & 3,52 \\
\hline Psidium cattleyanum Sabine & 0 & 1 & 0,79 & 2,70 & 5,56 & 3,02 \\
\hline Erythrina verna Vell. & 0 & 4 & 3,15 & 5,41 & 0,43 & 2,99 \\
\hline Calophyllum brasiliense Cambess. & 4 & 0 & 3,15 & 4,05 & 1,71 & 2,97 \\
\hline Sparattosperma leucanthum (Vell.) K. Schum. & 1 & 0 & 0,79 & 2,70 & 5,13 & 2,87 \\
\hline Lafoensia pacari A. St. Hil. & 1 & 0 & 0,79 & 6,76 & 0,85 & 2,80 \\
\hline Inga laurina (Sw.) Willd. & 5 & 2 & 5,51 & 1,35 & 1,28 & 2,72 \\
\hline Hymenaea courbaril L. & 1 & 0 & 0,79 & 6,76 & 0,43 & 2,66 \\
\hline Syzygium cumini (L.) Skeels & 1 & 0 & 0,79 & 5,41 & 0,85 & 2,35 \\
\hline Ocotea tabacifolia (Meisn.) Rohwer & 1 & 1 & 1,57 & 1,35 & 3,85 & 2,26 \\
\hline Pilocarpus pennatifolius Lem. & 1 & 0 & 0,79 & 1,35 & 4,27 & 2,14 \\
\hline Eugenia brasiliensis Lam. & 2 & 2 & 3,15 & 2,70 & 0,43 & 2,09 \\
\hline Handroanthus chrysothichum (Mart. ex A. DC.) Kuntze & 3 & 2 & 3,94 & 1,35 & 0,43 & 1,91 \\
\hline Nectandra megapotamica (Spreng.) Mez & 3 & 0 & 2,36 & 2,70 & 0,43 & 1,83 \\
\hline Psidium guajava $\mathrm{L}$. & 2 & 1 & 2,36 & 2,70 & 0,43 & 1,83 \\
\hline Andira anthelmia (Vell.) Macbride & 1 & 1 & 1,57 & 2,70 & 0,85 & 1,71 \\
\hline Senna multijuga (Rich.) H. S. & 0 & 2 & 1,57 & 1,35 & 1,28 & 1,40 \\
\hline Raulinoreitzia leptophlebia (B. L Rob.) & 1 & 0 & 0,79 & 2,70 & 0,43 & 1,31 \\
\hline Rapanea ferruginea (Ruiz \& Pav.) Mez & 1 & 1 & 1,57 & 1,35 & 0,43 & 1,12 \\
\hline Alibertia macrophylla Mart. & 1 & 0 & 0,79 & 1,35 & 0,85 & 1,00 \\
\hline Bauhinia fortificata Link & 1 & 0 & 0,79 & 1,35 & 0,85 & 1,00 \\
\hline Guazuma ulmifolia Lam. & 1 & 0 & 0,79 & 1,35 & 0,85 & 1,00 \\
\hline Maytenus ilicifolia Mart. ex Reissek & 1 & 0 & 0,79 & 1,35 & 0,85 & 1,00 \\
\hline Alchornea glandulosa Poepp. \& Endl. & 0 & 1 & 0,79 & 1,35 & 0,43 & 0,86 \\
\hline Anadenanthera colubrina (Vell.) Brenan & 1 & 0 & 0,79 & 1,35 & 0,43 & 0,86 \\
\hline Dodonea viscosa (L.) N. J. Jacq. & 1 & 0 & 0,79 & 1,35 & 0,43 & 0,86 \\
\hline Miconia cinerascens $\mathrm{Miq}$ & 1 & 0 & 0,79 & 1,35 & 0,43 & 0,86 \\
\hline Mimosa bimucronata (DC.) O. Kuntze. & 0 & 1 & 0,79 & 1,35 & 0,43 & 0,86 \\
\hline Piptadenia paniculata Benth & 1 & 0 & 0,79 & 1,35 & 0,43 & 0,86 \\
\hline Senna penducula (Hamb \& Bonpl) & 0 & 1 & 0,79 & 1,35 & 0,43 & 0,86 \\
\hline Solanum coavurana Vell. & 1 & 0 & 0,79 & 1,35 & 0,43 & 0,86 \\
\hline Solanum paniculatum $\mathrm{L}$. & 1 & 0 & 0,79 & 1,35 & 0,43 & 0,86 \\
\hline Machaerium stipitatum Vogel. & 1 & 0 & 0,79 & 1,35 & 0,00 & 0,71 \\
\hline
\end{tabular}

$\overline{\mathrm{NI}}$ (PL) - número de indivíduos plantados; NI (REG) - número de indivíduos regenerantes; DR - densidade relativa; FR - frequência relativa; DoR dominância relativa; VI -valor de importância. 
Tabela 3. Parâmetros fitossociológicos das espécies em área de restauração sobre bananal abandonado há 3 anos (Rest 3) no Parque Estadual do Rio Turvo, SP.

Table 3. Phytosociological parameters of the species in restoration areas on banana abandoned over 3 years (Rest 3 ) in the Rio Turvo State Park, SP, Brazil.

\begin{tabular}{|c|c|c|c|c|c|c|}
\hline ESPÉCIE & $\begin{array}{c}\text { NI } \\
(\mathrm{PL})\end{array}$ & $\begin{array}{c}\text { NI } \\
\text { (REG) }\end{array}$ & $\begin{array}{l}\text { DR } \\
(\%)\end{array}$ & $\begin{array}{c}\text { FR } \\
(\%)\end{array}$ & $\begin{array}{c}\text { DoR } \\
(\%) \\
\end{array}$ & $\begin{array}{r}\text { VI } \\
(\%)\end{array}$ \\
\hline Syagrus romanzoffiana (Cham.) Glassman & 4 & 0 & 1,43 & 0,82 & 26,04 & 9,43 \\
\hline Pipitadenia paniculata Benth & 1 & 2 & 1,08 & 3,28 & 14,64 & 6,33 \\
\hline Solanum mauritianum Scop. & 0 & 6 & 2,15 & 3,28 & 13,41 & 6,28 \\
\hline Eugenia uniflora DC. & 3 & 36 & 13,98 & 0,82 & 0,46 & 5,09 \\
\hline Pilocarpus pennatifolius Lem. & 2 & 17 & 6,81 & 4,92 & 1,85 & 4,53 \\
\hline Senna multijuga (Rich.) H. S. & 12 & 10 & 7,89 & 4,10 & 1,23 & 4,41 \\
\hline Trema micrantha (L.) Blume & 6 & 1 & 2,51 & 4,10 & 5,86 & 4,15 \\
\hline Cecropia hololeuca Miq. & 5 & 1 & 2,15 & 3,28 & 6,01 & 3,81 \\
\hline Lonchocarpus muehlbergianus Hassl. & 11 & 11 & 7,89 & 1,64 & 1,54 & 3,69 \\
\hline Piptadenia gonoacantha (Mart.) J. F. Macbr. & 1 & 17 & 6,45 & 2,46 & 0,31 & 3,07 \\
\hline Schizolobium parahyba (Vell.) & 1 & 0 & 0,36 & 0,82 & 6,78 & 2,65 \\
\hline Inga laurina (Sw.) Willd. & 11 & 2 & 4,66 & 1,64 & 0,31 & 2,20 \\
\hline Maytenus ilicifolia Mart. ex Reissek & 4 & 4 & 2,87 & 3,28 & 0,15 & 2,10 \\
\hline Lithraea molleoides (Vell.) Engl. & 0 & 10 & 3,58 & 2,46 & 0,15 & 2,07 \\
\hline Rapanea ferruginea (Ruiz \& Pav.) Mez & 4 & 2 & 2,15 & 2,46 & 1,54 & 2,05 \\
\hline Psidium guajava $\mathrm{L}$. & 3 & 2 & 1,79 & 3,28 & 0,77 & 1,95 \\
\hline Bombacopsis glabra (Pasq.) A. Robyns & 3 & 0 & 1,08 & 4,10 & 0,31 & 1,83 \\
\hline Eugenia malaccensis & 0 & 1 & 0,36 & 4,92 & 0,15 & 1,81 \\
\hline Miconia brunnea DC. & 8 & 4 & 4,30 & 0,82 & 0,15 & 1,76 \\
\hline Campomanesia pubescens (DC.) O. Berg & 0 & 3 & 1,43 & 3,28 & 0,15 & 1,62 \\
\hline Vernonia polyanthes Less. & 0 & 2 & 0,72 & 1,64 & 2,47 & 1,61 \\
\hline Inga cylindrica Mart. & 2 & 2 & 1,43 & 2,46 & 0,31 & 1,40 \\
\hline Jacaranda macrantha Cham & 2 & & 0,72 & 3,28 & 0,15 & 1,38 \\
\hline Pothomorphe umbellata & 0 & 5 & 1,79 & 1,64 & 0,62 & 1,35 \\
\hline Ingá edulis Mart. & 4 & 0 & 1,43 & 2,46 & 0,15 & 1,35 \\
\hline Strychnos pseudoquina A.St.-Hil. & 3 & 0 & 1,08 & 1,64 & 1,23 & 1,32 \\
\hline Lonchocarpus guilleminianus (Tul.) Malme & 0 & 4 & 1,43 & 0,82 & 1,39 & 1,21 \\
\hline Gochnatia pulchra Cabrera & 1 & 0 & 0,36 & 1,64 & 1,54 & 1,18 \\
\hline Solanum paniculatum $\mathrm{L}$. & 0 & 1 & 0,36 & 0,82 & 2,31 & 1,16 \\
\hline Senna pandulata (Humb \& Bonpl) & 0 & 3 & 1,08 & 1,64 & 0,62 & 1,11 \\
\hline Inga marginata Willd. & 2 & 2 & 1,43 & 1,64 & 0,15 & 1,08 \\
\hline Morus nigra $\mathrm{L}$. & 0 & 4 & 1,43 & 1,64 & 0,15 & 1,08 \\
\hline Machaerium stipitatum Vogel. & 0 & 1 & 0,36 & 0,82 & 2,00 & 1,06 \\
\hline Erythrina verna Vell. & 3 & 0 & 1,08 & 1,64 & 0,31 & 1,01 \\
\hline Ocotea tabacifolia (Meisn.) Mez & 1 & 0 & 0,36 & 2,46 & 0,15 & 0,99 \\
\hline Swartzia langsdorffii Raddi & 0 & 5 & 1,79 & 0,82 & 0,15 & 0,92 \\
\hline Anadenanthera colubrina (Vell.) Brenan & 0 & 2 & 0,72 & 1,64 & 0,31 & 0,89 \\
\hline Cryptocarya aschersoniana $\mathrm{Mez}$ & 0 & 1 & 0,36 & 1,64 & 0,15 & 0,72 \\
\hline Eriobotrya japonica Lindl. & 0 & 1 & 0,36 & 1,64 & 0,15 & 0,72 \\
\hline Nectandra megapotamica (Spreng.) Mez & 2 & 0 & 0,72 & 0,82 & 0,46 & 0,67 \\
\hline Calyptranthes grandifolia $\mathrm{O}$. Berg. & 0 & 1 & 0,36 & 0,82 & 0,62 & 0,60 \\
\hline Solanum coavurana Vell. & 0 & 1 & 0,36 & 0,82 & 0,62 & 0,60 \\
\hline Cupania oblongifolia Mart. & 0 & 2 & 0,72 & 0,82 & 0,15 & 0,56 \\
\hline Eugenia dura Merr. & 0 & 2 & 0,72 & 0,82 & 0,15 & 0,56 \\
\hline
\end{tabular}

Continua... 
Tabela 3. Continuação.

\begin{tabular}{|c|c|c|c|c|c|c|}
\hline ESPÉCIE & $\begin{array}{c}\text { NI } \\
(\mathrm{PL})\end{array}$ & $\begin{array}{c}\text { NI } \\
\text { (REG) }\end{array}$ & $\begin{array}{l}\text { DR } \\
\text { (\%) }\end{array}$ & $\begin{array}{l}\text { FR } \\
(\%)\end{array}$ & $\begin{array}{c}\text { DoR } \\
\text { (\%) }\end{array}$ & $\begin{array}{r}\text { VI } \\
(\%)\end{array}$ \\
\hline Machaerium aculeatum Raddi & 1 & 3 & 0,72 & 0,82 & 0,15 & 0,56 \\
\hline Bauhinia forficata Link & 0 & 1 & 0,36 & 0,82 & 0,31 & 0,50 \\
\hline Gochnatia polymorpha (Less.) Cabrera & 0 & 1 & 0,36 & 0,82 & 0,31 & 0,50 \\
\hline Alchornea glandulosa Poepp. \& Endl. & 1 & 0 & 0,36 & 0,82 & 0,15 & 0,44 \\
\hline Bathysa australis (A. St.-Hil.) K.Schum & 0 & 1 & 0,36 & 0,82 & 0,15 & 0,44 \\
\hline Citrus sinensis Osbeck & 0 & 1 & 0,36 & 0,82 & 0,15 & 0,44 \\
\hline Copaifera langsdorffii Desf. & 0 & 1 & 0,36 & 0,82 & 0,15 & 0,44 \\
\hline Ocotea paranapiacabensis Coe-Teixeira & 1 & 0 & 0,36 & 0,82 & 0,15 & 0,44 \\
\hline Peltophorum dubium (Spreng.) Taub. & 0 & 1 & 0,36 & 0,82 & 0,15 & 0,44 \\
\hline Vismia brasiliensis Choisy & 0 & 1 & 0,36 & 0,82 & 0,15 & 0,44 \\
\hline
\end{tabular}

NI (PL) - número de indivíduos plantados; NI (REG) - número de indivíduos regenerantes; DR - densidade relativa; FR - frequência relativa; DoR - dominância relativa; VI -valor de importância.

A comparação pelo teste Kruskall-Wallis (Tabela 4) mostra que a área Rest 3 apresentou área basal e densidade superior $(\mathrm{p} \leq 0,05)$ à Rest 5 .

Entre as espécies que foram plantadas há 3 anos (Rest 3), os maiores valores de altura foram registrados para Trema micrantha (L.) $(15,0 \mathrm{~m})$, Senna multijuga $(11,8 \mathrm{~m})$, Cecropia hololeuca $(9,5 \mathrm{~m})$, Inga laurina (Sw.) $(9,0 \mathrm{~m})$ e Schizolobium parahyba $(7,5 \mathrm{~m})$. Entre aquelas plantadas há 5 anos (Rest 5), também se destacaram, com os maiores valores em altura, $I$. laurina ( $8,0 \mathrm{~m})$, além das espécies Guazuma ulmifolia (10,0 m), Pilocarpus pennatifolius (4,5 m), Dodonea viscosa (4,5 m) e Campomanesia guazumifolia $(4,5 \mathrm{~m})$.
A análise dos indivíduos plantados e regenerantes por classe de altura na Rest 3 mostrou maiores percentuais de indivíduos nas classes I, II e III, enquanto que na Rest 5 , os maiores percentuais concentraram-se nas classes II, III e IV (Figuras 4a e 4b).

$\mathrm{Na}$ Rest 5 , as espécies regenerantes das maiores classes de altura foram Cecropia hololeuca Miq. (10,0 m), Mimosa bimucronata $(8,0 \mathrm{~m})$, Croton urucurana $(6,5$ $\mathrm{m})$ e Syagrus romanzoffiana $(6,0 \mathrm{~m})$, enquanto na Rest 3 pertencem às maiores classes de altura Piptadenia gonoacantha $(10,0 \mathrm{~m})$, Vernonia polyanthes $(9,5 \mathrm{~m})$, S. multijuga $(9,0 \mathrm{~m})$, Solanum paniculatum $(14,0 \mathrm{~m}) \mathrm{e}$ Machaerium aculeatum $(7,0 \mathrm{~m})$.
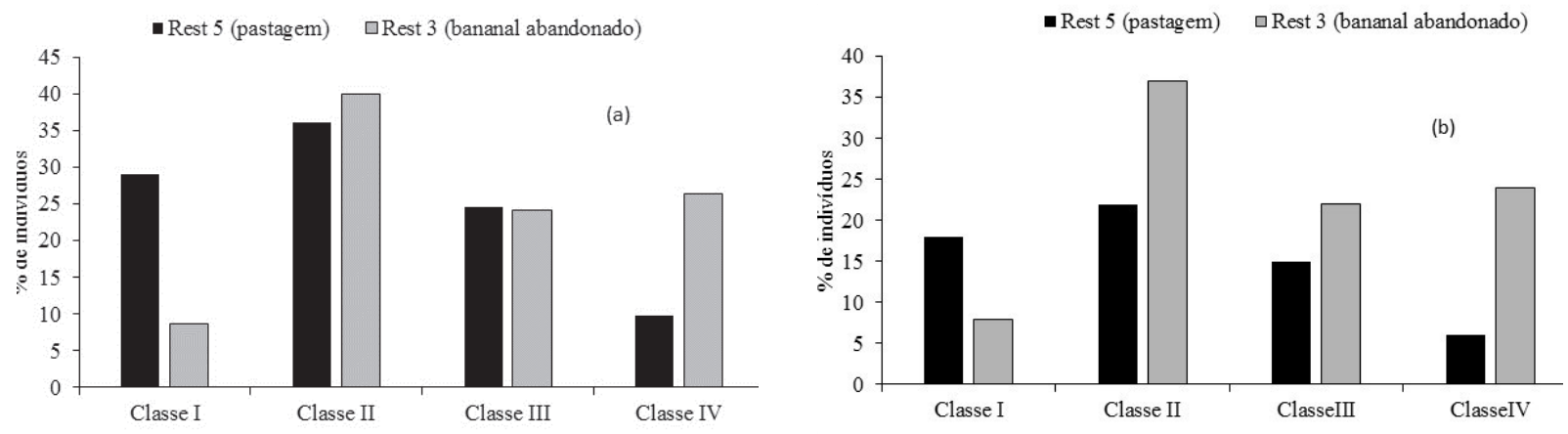

Figura 4. Distribuição das alturas (h) dos indivíduos plantados (a) e regenerantes (b) amostrados nas áreas de restauração: Classe I - 0,5 $\mathrm{m} \leq \mathrm{h} \leq 0,99 \mathrm{~m}$; Classe II - $1 \mathrm{~m} \leq \mathrm{h} \leq 2,99 \mathrm{~m}$; Classe III - 3,0 $\mathrm{m} \leq \mathrm{h} \leq 4,99 \mathrm{~m}$; Classe IV: $\mathrm{h} \geq 5,0 \mathrm{~m}$.. Rest $5=$ área de pastagem em processo de restauração há cinco anos; Rest 3 = área de bananal abandonado há três anos em processo de restauração.

Figure 4. Height $(\mathrm{h})$ distribution of the regenerating individuals in the forest fragments. Class I - 0,5 $\mathrm{m} \leq \mathrm{h} \leq 0,99 \mathrm{~m}$; Class II $1 \mathrm{~m} \leq \mathrm{h} \leq 2,99 \mathrm{~m}$; Class III $-3,0 \mathrm{~m} \leq \mathrm{h} \leq 4,99 \mathrm{~m}$; Class IV: $\mathrm{h} \geq 5,0 \mathrm{~m}$. Rest 5 = restoration area over five years, on a degraded area of grazing land; Rest $3=$ restoration area over three years, on an abandoned banana plantation. 
Tabela 4. Parâmetros estruturais para as duas áreas avaliadas.

Table 4. Structural parameters for the two evaluated areas.

\begin{tabular}{cccccc}
\hline & $\begin{array}{c}\text { Área basal } \\
\left(\mathbf{m}^{\mathbf{2}} \mathbf{h a} \mathbf{a}^{-1}\right)\end{array}$ & $\begin{array}{c}\text { Altura Média } \\
\mathbf{( m )}\end{array}$ & $\begin{array}{c}\text { Densidade } \\
\left(\mathbf{I n d ~ h a} \mathbf{~}^{-\mathbf{1}}\right)\end{array}$ & $\begin{array}{c}\text { Índice de Shannon } \\
(\mathbf{H})\end{array}$ & $\begin{array}{c}\text { Equabilidade } \\
(\mathbf{J})\end{array}$ \\
\hline Rest 5 & $9,51 \mathrm{~b}$ & $2,24 \mathrm{~b}$ & $2.117 \mathrm{~b}$ & 3,09 & 0,51 \\
Rest 3 & $12,86 \mathrm{a}$ & $3,93 \mathrm{a}$ & $4.650 \mathrm{a}$ & 3,43 & 0,50 \\
\hline
\end{tabular}

Médias seguidas por letras iguais não diferem entre si pelo teste de Kruskall-Wallis $(\mathrm{p} \leq 0,05)$. Rest $5=$ área de pastagem em processo de restauração há cinco anos; Rest 3 = área de bananal abandonado há três anos em processo de restauração.

\section{Discussão}

Os solos da área de estudo são considerados ácidos, sendo a acidez mais acentuada na área de pastagem em processo de restauração há cinco anos (Rest 5). O pH mais elevado na área de bananal abandonado há três anos em processo de restauração (Rest 3) parece estar relacionado aos teores mais elevados de $\mathrm{Ca}$ e $\mathrm{Mg}$. As diferenças nos teores de $\mathrm{Ca}$ e $\mathrm{Mg}$ também se refletiram na maior soma de bases e saturação por bases na Rest 3 . Os solos amostrados na Rest 5 são considerados pobres em $\mathrm{P}$, com valores abaixo de $10 \mathrm{mg} \mathrm{dm}^{-3}$. Os teores de matéria orgânica situam-se dentro da faixa para solos de textura média (16 e $30 \mathrm{~g} \mathrm{dm}^{-3}$ ). Possivelmente, o pH mais elevado (Tabela 1) observado na área Rest 3, comparando-se com a área Rest 5, seja o efeito residual da adubação realizada anteriormente na área. Por outro lado, a maior acidez do solo cujo uso anterior foi pastagem (Rest 5) pode ter reflexos no estabelecimento da vegetação, já que sob essa condição a maioria das espécies sofre redução no crescimento (Holl et al., 1999). Para Mendes et al. (2019), a fertilidade do solo é um fator limitante ao desenvolvimento da vegetação em pastagens, especialmente em áreas com longo histórico de uso e sem adoção de um manejo adequado. Sobanski \& Marques (2014), avaliando processos de restauração em áreas de pastagem, concluíram que as condições edáficas do solo (classe de solo, fertilidade, condições físicas) exercem tanta influência negativa no crescimento e sobrevivência de espécies arbóreas da Mata Atlântica quanto a espécie de gramínea utilizada.

Pelos valores de saturação de bases (V\%), os solos das duas áreas avaliadas são considerados eutróficos, mas os solos da Rest 3 apresentaram saturação de $83 \%$, refletindo a constante adubação dos bananais antes da área ser abandonada, o que poderia influenciar positivamente no estabelecimento das espécies plantadas na restauração. Para Lambers et al. (2008), a fertilidade do solo também influencia sobremaneira o sucesso de restauração florestal, uma vez que o desenvolvimento das plantas está diretamente relacionado com a disponibilidade de nutrientes no sistema.

As proporções entre pioneiras e não pioneiras plantadas nas duas áreas avaliadas $(25 \% \mathrm{P}$ e $75 \% \mathrm{NP}$ na Rest 5 e 30\% P e 70\% NP na Rest 3) são decorrentes do método utilizado para o plantio (linhas alternadas de pioneiras e não pioneiras). Esses percentuais encontramse abaixo do estabelecido pela Resolução SMA/SP $n^{\circ}$ 32/2014 (São Paulo, 2014), que recomenda o plantio de um mínimo de $40 \%$ de espécies para cada grupo ecológico em processos de restauração.

Os maiores números de indivíduos e riqueza de espécies regenerantes (Figura 3), de acordo com a Resolução SMA/SP no 32/2014 (São Paulo, 2014), são indicadores de que a restauração está ocorrendo com maior sucesso (aumento de diversidade) na Rest $3 \mathrm{em}$ comparação com a Rest 5, considerando ainda que a área está em regeneração a apenas três anos. Quando esses valores são comparados com os índices recomendados pelo Protocolo de monitoramento para programas e projetos de restauração florestal (Rodrigues et al., 2013) para regeneração do sub-bosque aos 5 anos após o plantio (> 20 spp. - aceitável; entre 10 e 20 spp. - preocupante; abaixo de $10 \mathrm{spp}$.- requer correções), verifica-se que a Rest 3 já alcançou esses valores, enquanto na Rest 5 o número de espécies regenerantes é considerado preocupante.

Os maiores percentuais de espécies pioneiras entre as regenerantes encontrados na Rest 5 seguiram o comportamento esperado para reflorestamentos em que se consorciam diferentes grupos ecológicos. As espécies pioneiras, geralmente, apresentam rápido crescimento, rápido crescimento, agressividade e boa sobrevivência à pleno sol, permitindo um rápido recobrimento de uma área degradada por essas espécies, que preparariam assim as condições para o desenvolvimento das espécies 
clímaces que localmente formariam a floresta definitiva (Rodrigues et al., 2009). O mesmo não ocorreu para a Rest 3, onde o percentual de regenerantes não pioneiras foi maior que o das pioneiras, provavelmente reflexo de condições mais favoráveis de facilitação. Estas condições representam, dentre outras, redução da luminosidade, que desfavorece a germinação de sementes de espécies ruderais do banco do solo (Figueiredo et al., 2014), e temperaturas mais baixas, para evitar altas taxas de respiração por essas plantas nos estágios iniciais de vida (Farias et al., 2012).

As maiores proporções de espécies zoocóricas encontradas nas duas áreas, principalmente entre os regenerantes, são indicadores importantes no processo de restauração. A elevada proporção de espécies zoocóricas entre os indivíduos regenerantes sob plantios tem sido considerada como indicador de sucesso da restauração, pois demonstra que a fauna dispersora está atuante, colaborando para a superação dos filtros que dificultam a chegada de propágulos à comunidade em formação (Suganuma et al., 2013). Ziparro et al. (2005), em levantamento realizado em remanescente de Mata Atlântica no Parque Estadual Intervales - Base Saibadela, identificaram a síndrome zoocórica em $80,7 \%$ das 419 espécies. Em trabalhos realizados em outras localidades de Mata Atlântica, esse tipo de dispersão englobou 89,2\% das espécies arbustivo-arbóreas (Talora \& Morellato, 2000). Esses valores mostram a importância da fauna para as espécies vegetais e o alto número de interações interespecíficas (conectância), abordando apenas a dispersão de sementes existentes na Floresta Atlântica (Ziparro et al., 2005).

A maior proporção de indivíduos pertencentes à família Fabaceae (Figura 2) encontrados nesse estudo diferem de outros estudos da Mata Atlântica no Sudeste e Sul do Brasil, em que Myrtaceae aparece como a família com maior riqueza, seguida de Fabaceae. Rochelle et al. (2011) realizaram uma revisão de 28 desses estudos e descobriram que a riqueza foi maior para Myrtaceae em 21 deles, enquanto Fabaceae foi maior em apenas quatro. A maioria dos estudos realizados em Floresta Ombrófila Densa no Estado de São Paulo mostram Myrtaceae como a família mais rica, geralmente seguida por Fabaceae, Rubiaceae, Lauraceae, Melastomataceae e Euphorbiaceae (Urbanetz et al., 2010).

As espécies da família Fabaceae têm sido utilizadas como uma das alternativas para a recuperação de áreas degradadas e, ecologicamente, destacam-se devido à sua ampla ocorrência e adaptação (Silva \& Melo, 2013). Além disso, possuem capacidade de se associarem simbioticamente às bactérias fixadoras de nitrogênio que, juntamente com o fósforo, são os nutrientes que mais limitam o estabelecimento e o desenvolvimento vegetal. A fixação biológica de nitrogênio apresenta grande importância para programas de recuperação de solos degradados, geralmente deficientes desse elemento, em virtude da intensa perda de matéria orgânica. Tais características das Fabaceae favorecem a potencialidade do seu aproveitamento agrícola e silvicultural (Nogueira et al., 2012). Segundo Rochelle et al. (2011), mirtáceas têm grande importância florística em toda costa brasileira, devido à elevada riqueza de suas espécies lenhosas. A produção de frutos e a facilidade de estabelecimento também são características favoráveis para os processos de restauração.

A diversidade que compõem a regeneração natural nas áreas em processo de restauração pode representar um dos descritores mais eficentes na avaliação do sucesso de iniciativas como essas, além de ser um excelente indicador das ações de manejo necessárias para melhorar os resultados e garantir a sustentabilidade das áreas restauradas (Souza, 2014; Reis et al., 2014). O percentual de espécies plantadas e regenerantes foi significativamente diferente para as duas condições avaliadas, com a Rest 3 apresentando o maior percentual de regenerantes (Figura 3). O histórico de uso da área, refletido na maior fertilidade inicial, provavelmente favoreceu o desenvolvimento dos regenerantes. $\mathrm{O}$ atributo regeneração natural como indicador do processo de restauração pode estar relacionado às condições propícias criadas para o estabelecimento de plântulas através do favorecimento de microclima, fechamento do dossel (dependendo das espécies plantadas), melhorias na fertilidade do solo pela formação de camadas de serapilheira e húmus, atratividade a agentes dispersores e supressão de espécies invasoras (Engel \& Parrota, 2008). Do mesmo modo, o menor número e riqueza de espécies regenerantes encontrados na Rest 5 é, possivelmente, consequência da baixa fertilidade inicial do solo e do manejo da gramínea praticado ao longo do processo de restauração. Cheung et al. (2009) constataram que em locais onde ocorre alta quantidade de biomassa de gramínea exótica, as espécies mais exigentes são impedidas de se estabelecerem, tendo como consequência uma menor riqueza, densidade e volume de espécies arbóreas. 
A presença de espécies colonizadoras (não plantadas) na regeneração natural encontradas em proporções cinco vezes maiores na Rest 3, quando comparadas à Rest 5 , bem como a caracterização da síndrome de dispersão zoocórica dessas espécies, são indicadores dos processos ecológicos que podem garantir a entrada e estabecimento de novas espécies na área restaurada, recuperando a biodiversidade que é o requisito principal para a sustentabilidade desses ambientes. Provavelmente, essas espécies que não foram plantadas e estão colonizando a área vieram das florestas próximas. Isso indica um fluxo de dispersão de sementes e um grande potencial de recuperação da trajetória sucessional das áreas em restauração (Suganuma et al., 2014). Para Silva (2003) e Jordano et al. (2006), esses resultados refletem a atuação da fauna de dispersores que foram atraídos para a área restaurada por algum motivo (abrigo, alimento, corredores, etc.), dispersores esses oriundos de áreas naturais do entorno, dando uma boa indicação do papel da restauração vegetal no resgate da fauna local e da atuação dessas áreas restauradas como corredores ecológicos na paisagem regional.

Os maiores percentuais de regenerantes pertencentes ao grupo das não pioneiras na Rest 3 são também indicativos de processos ecológicos favoráveis ao avanço da restauração. De acordo com Melo \& Durigan (2007), esses resultados sugerem que as árvores plantadas ainda não atingiram estágio reprodutivo, mas proporcionaram habitats atrativos para animais dispersores de sementes.

Os diferentes valores de importância (VI) para as espécies descritas nesse estudo foram influenciados pela área basal e número de indivíduos. Para Oliveira et al. (2006), a predominância de algumas espécies mais adaptadas no início dos processos de sucessão é comum e há uma tendência de equilíbrio na distribuição das espécies na comunidade, com o aumento do tempo das restaurações. Destaca-se que, entre as espécies com maior VI na Rest 3, 60\% pertence ao grupo das pioneiras, enquanto na Rest 5 70\% das espécies com maior VI pertencem ao grupo das não pioneiras. Informações sobre espécies com maior VI são importantes por demonstrarem a afinidade florística entre os fragmentos florestais da região, podendo ser útil na escolha das espécies florestais para projetos de recuperação de áreas degradadas, bem como ratifica o uso potencial dessas espécies em planos de manejo e de conservação florestal (Ferreira Junior et al., 2007).
Os valores de área basal determinados nesse estudo para a Rest $3\left(12,86 \mathrm{~m}^{2} \mathrm{ha}^{-1}\right)$, são próximos aos registrados por Melo \& Durigan (2007) em matas ciliares nativas e plantadas em diferentes idades no Vale do Paranapanema, SP (área basal aos três anos entre 15,1 e $16,2 \mathrm{~m}^{2} \mathrm{ha}^{-1}$ ).

As diferenças significativas entre os valores de densidade de indivíduos para as duas áreas avaliadas, bem como o número de indivíduos por hectare quatro vezes maior na Rest 3, confirmam um melhor desenvolvimento da restauração sobre bananal abandonado, onde os fatores fertilidade do solo e menor competição com gramíneas foram diferenciais para o resultado nesse período de tempo. Em pastagens abandonadas, alguns fatores são apontados como as principais barreiras para o sucesso da regeneração e recuperação de ambientes florestais: densidade de gramíneas, chegada de sementes (recrutamento de novos indivíduos e espécies) e condições microclimáticas que possibilitem a germinação das sementes e o estabelecimento das plântulas (Holl et al., 2000).

Martins (1991) afirma que os valores de índice de diversidade de Shannon encontrados com maior frequência na Mata Atlântica variam de 3,8 a 5,8. Nesse estudo, os valores aproximaram-se do mínimo, o que é decorrente das áreas se encontrarem em fase inicial de restauração. Os menores valores calculados para a Rest 5 $(3,09)$ são reflexo do uso anterior da área com pastagem abandonada, pois esse tipo de sistema representa um fator de degradação e, consequentemente, dificulta o estabelecimento de espécies arbóreas. A baixa riqueza e abundância de plântulas estabelecidas nessa área podem decorrer da dominância do ambiente por plantas herbáceas, pois quando gramíneas e outras herbáceas dominam um terreno, reduzem os níveis de umidade e nutrientes do solo, impondo um ambiente desfavorável para o estabelecimento da maioria das plantas lenhosas (Rhoades et al., 1998).

Os índices de equabilidade (J') também refletem o início do processo de restauração das duas áreas, sendo que se espera um aumento dos valores ao longo de tempo e uma maior uniformidade na distribuição das espécies, a exemplo do J' $=0,66$ descrito por Moura \& Mantovani (2017) em área de regeneração natural de 8 anos sobre bananal abandonado em Floresta Ombrófila Densa no Vale do Ribeira.

A distribuição das espécies nas diferentes classes de altura na Rest 3 (Figura 4) revela a recuperação 
da vegetação. De acordo com Silva et al. (2015), a estabilidade local das espécies se confirma com o surgimento de indivíduos regenerantes nas classes de maior tamanho. Segundo Hosokawa et al. (1998), as espécies asseguram sua representatividade na estrutura da comunidade florestal quando estão presentes em todos os estratos. As que não conseguem acompanhar esta regra tendem a desaparecer, seja porque não se reproduzem, seja porque não conseguem se estabelecer nas condições prevalecentes.

O mesmo fenômeno não ocorre na área de restauração sobre pastagem, pois as intempéries climáticas e a exposição solar favorecem o domínio de espécies pioneiras. Além disso, é comum a inibição do processo de regeneração natural na presença de gramíneas, reduzindo a distribuição de nutrientes e acesso à luminosidade, ocasionando uma barreira física ao processo de germinação e estabelecimento de plântulas (Hoffmann \& Haridasan, 2008).

\section{Agradecimentos}

Ao Instituto Florestal e à Fundação Florestal pelo apoio logístico, essencial para o desenvolvimento do trabalho.

\section{Conclusões}

A restauração está mais avançada em área sobre bananal abandonado há três anos, o que foi evidenciado pelos maiores números de indivíduos, famílias, espécies, percentual de regenerantes e áreas basais.

Os parâmetros fitossociológicos refletem áreas em processos iniciais de sucessão secundária, onde o uso anterior, com adubações periódicas, na ausência de gramíneas incorreu na composição de um ambiente mais favorável para o desenvolvimento das espécies plantadas e estabelecimento de propágulos.

\section{Nota de final de texto}

${ }^{1}$ Informação coletada in loco na visita ao PERT na fase de prospecção das áreas do estudo em abril de 2016.

\section{Referências}

Álvares C. A. et al. Köppen's climate classification map for Brazil. Meteorologische Zeitschrift, v. 22, n. 6, p. 711-728, 2013. https:// doi.org/10.1127/0941-2948/2013/0507.
APG IV. Angiosperm Phylogeny Group. An update of the Angiosperm Phylogeny Group classification for the orders and families of flowering plants: APG IV. Botanical Journal of the Linnean Society, v. 181, n. 1, p. 1-20, 2016. https://doi.org/10.1111/ boj.12385.

Barbosa, L. M. et al. Lista de espécies indicadas para restauração ecológica para diversas regiões do Estado de São Paulo. São Paulo: Instituto de Botânica, 2017. 344 p.

Brancalion, P. H. S. et al. Plantio de árvores nativas brasileiras fundamentado na sucessão florestal. In: Rodrigues, R. R. et al. (org.) Pacto para a restauração ecológica da Mata Atlântica: referencial dos conceitos e ações de restauração florestal. São Paulo: Instituto BioAtlântica, 2009. p. 14-23.

Brasil. Ministério do Meio Ambiente. Avaliação e ações prioritárias para a conservação da biodiversidade da Mata Atlântica e Campos Sulinos. Brasília, DF, 2000. 40 p.

Brazil Flora G. Brazilian Flora 2020 project $=$ Projeto Flora do Brasil 2020. Version 393.280. Rio de Janeiro: Instituto de Pesquisas Jardim Botânico do Rio de Janeiro, 2021. Checklist dataset: https:// doi.org/10.15468/1mtkaw.

Chazdon, R. L. Chance and determinism in tropical forestsuccession. In: Carson, W. P. \& Schnitzer, S. A. (ed.). Tropical forest community ecology. Malden: Blackwell, 2008. p. 384-408.

Cheung, K. C. et al. Relação entre a presença de vegetação herbácea e a regeneração natural de espécies lenhosas em pastagens abandonadas na Floresta Ombrófila Densa do Sul do Brasil. Acta Botanica Brasilica, v. 23, p. 1048-1056, 2009.

Classen, M. E. C. (org.). Manual de métodos de análise de solo. 2. ed. Rio de Janeiro: EMBRAPA-CNPS, 1997. 212 p. (EMBRAPACNPS. Documentos, 1).

Engel, V. L. \& Parrotta, J. A. Definindo a restauração ecológica: tendências e perspectivas mundiais. In: Kageyama, P. Y. et al. (org.). Restauração ecológica de ecossistemas naturais. Botucatu: FEPAF, 2008. p. 3-25.

Farias, L. N. et al. Parâmetros microclimáticos e atributos químicos e físicos do solo em área de preservação permanente situada na cabeceira do rio São Lourenço, Campo Verde - MT. Engenharia Ambiental, v. 9, n. 1, p. 83-99, 2012.

Ferreira Júnior, W. G. et al. Composição florística da vegetação arbórea de um trecho de Floresta Estacional Semidecídua em Viçosa, Minas Gerais, e espécies de maior ocorrência na região. Revista Árvore, v. 31, n. 6, p. 1131-1143, 2007.

Figueiredo, P. H. A. et al. Germinação ex-situ do banco de sementes do solo de capoeira em restauração florestal espontânea a partir do manejo do sombreamento. Scientia Forestalis, v. 42, n. 101, p. 69-80, 2014.

Fonseca, D. A. et al. Avaliação da regeneração natural em área de restauração ecológica e mata ciliar de referência. Ciência Florestal, v. 27, n. 2, p. 521-534, 2017. http://dx.doi.org/10.5902/1980509827733.

Hoffmann, W. A.; Haridasan, M. The invase grass, Melinis minuliflora inibitis tree regeneration in a neotropical savanna. Austral Ecology, v. 33. n. 1, p. 29-36, 2008. 
Holl, K. D. et al. Tropical montane forest restoration in Costa Rica: overcoming barriers to dispersal and establishment. Restoration Ecology, v. 8, p. 339-349, 2000.

Holl, K. D. Factors limiting tropical rain forest regeneration in abandoned pasture: seed rain, seed germination, microclimate and soil. Biotropica, v. 31, p. 229-242, 1999.

Holl, K. D. Restoring from the botton up. Science, v. 355, n. 6324, p. $455-456,2017$.

Holl, K. D. Restoring tropical forest. Nature Education Knowledge, v. 4, n. 4, 2013. Disponível em: https://www.nature.com/scitable/ knowledge/library/restoring-tropical-forest-97756726. Acesso em: 10 mar. 2019.

Hosokawa, R. T. et al. Introdução ao manejo e economia florestal. Curitiba: Ed. da UFPR, 1998.

IBGE. Instituto Brasileiro de Geografia e Estatística. Manual técnico da vegetação brasileira. 2. ed. Rio de Janeiro, 2012. 275 p. (Série manuais técnicos em Geociências, 1).

Jordano, P. et al. Ligando frugivoria e dispersão de sementes à Biologia da Conservação. In: Rocha, C. F. D. et al. (ed.). Biologia da conservação: essências. São Paulo: Rima, 2006. p. 411-436.

Köppen, W. \& Geiger, R. Klimate der Erde. Gotha: Verlag Justus Perthes, 1928.

Kronka, F. J. N. et al. Inventário florestal da vegetação natural do Estado de São Paulo: regiões administrativas de São José dos Campos (Litoral), Baixada Santista e Registro. São Paulo: Imprensa Oficial, 2007. 140 p.

Lambers, H. et al. Plant nutrient-acquisition strategies change with soil age. Trends in Ecology \& Evolution, v. 23, p. 95-103, 2008.

Martins F. R. Estrutura de uma floresta mesófila. Campinas: Ed. UNICAMP, 1991.

Melo, A. C. G. \& Durigan, G. Structural evolution of planted riparian forests in the Medium Paranapanema Valley, SP, Brazil. Scientia Florestalis, v. 1, n. 73, p. 101-111, 2007.

Mônico, A. C. Alternativas para restauração florestal de pastagens. 2019. 180 f. Tese (Doutorado em Ciências) - Universidade de São Paulo, Piracicaba.

Moura, C. \& Mantovani, W. Regeneração natural da floresta ombrófila densa após oito anos de abandono de atividades agrícolas em Miracatu, Vale do Ribeira, SP. Revista do Instituto Florestal, v. 29 n. 1 p. 91-119, 2017. http://dx.doi.org/10.24278/21785031.201729106 .

Mueller-Dombois, D. \& Ellenberg, H. Aims and methods of vegetation ecology. New York: John Willey \& Sons, 1974.

Nalon, M. A. et al. Meio físico e aspectos da fragmentação da vegetação. In: Rodrigues, R. R. \& Bononi, V. L. R. (org.). Diretrizes para a restauração da biodiversidade no Estado de São Paulo. São Paulo: Instituto de Botânica: Imprensa Oficial, 2008. p. 14-21.

Nogueira, F. C. B. et al. Efeito da temperatura e luz na germinação de sementes de Luetzelburgia auriculata (Alemão) Ducke - Fabaceae. Acta Botanica Brasilica, v. 6, n. 4, p. 72-778, 2012.
Oliveira, L. C. et al. Exploração florestal e eficiência dos tratamentos silviculturais realizados em uma área de 136 ha na Floresta Nacional do Tapajós, Belterra - Pará. Revista de Ciências Agrárias, v. 46, p. 195-213, 2006.

Reis, D. N. et al. Indicadores preliminares para avaliação da restauração em reflorestamentos de ambientes ciliares. Pesquisa Florestal Brasileira. v. 34, p. 375-389, 2014. http://dx.doi. org/10.4336/2014.pfb.34.80.757.

Rhoades, C. C. et al. Effect of pasture trees on soil nitrogen and organic matter: implications for tropical montane forest restoration. Restoration Ecology, v. 6, p. 262-270, 1998.

Rochelle, A. L. C. et al. Florística e estrutura de um trecho de Floresta Ombrófila Densa Atlântica Submontana no Parque Estadual da Serra do Mar, em Ubatuba/SP, Brasil. Biota Neotropica, n. 2, p. 337-346, 2011.

Rodrigues, R. R. et al. Pacto pela restauração da Mata Atlântica: referencial dos conceitos e ações de restauração florestal. São Paulo: LERF/ESALQ: Instituto BioAtlântica, 2009. 264 p.

Rodrigues, R. R. et al. Pacto pela restauração da Mata Atlântica: referencial dos conceitos e ações de restauração florestal. São Paulo: LERF/ESALQ, 2011.

Rodrigues, R. R. et al. (ed.). Protocolo de monitoramento para programas e projetos de restauração florestal. Piracicaba: ESALQ, 2013.

São Paulo (Estado). Resolução SMA no 32 de 3 de abril de 2014. Estabelece as orientações, diretrizes e critérios sobre restauração ecológica no Estado de São Paulo, e dá providências correlatas. Diário Oficial do Estado, São Paulo, Seção 1, p. 36-37, 5 abr. 2014. Disponível em: https://smastr16.blob.core.windows.net/ legislacao/2016/12/Resolu\%C3\%A7\%C3\%A3o-SMA-032-2014-a. pdf. Acesso em: 10 dez. 2014.

São Paulo (Estado). Secretaria do Meio Ambiente. Instituto Florestal. Mapa pedológico do Estado de São Paulo: revisado e ampliado. São Paulo, 2017. 118 p.

Shannon, C. E. A mathematical theory of communication. Bell SystemTechnical Journal, v. 27, p. 379-42, 1948.

Shepherd, G. J. Fitopac versão 2.1: manual do usuário. Campinas: Departamento de Botânica da Universidade Estadual de Campinas, 2010. Disponível em: http://pedroeisenlohr.webnode.com.br/fitopac/. Acesso em: 15 nov. 2019.

Silva, L. S. et al. Florística, estrutura e sucessão ecológica de um remanescente de mata ciliar na bacia do rio Gurguéia-PI. Nativa, v. 3, n. 3, p. 156-164, 2015.

Silva, S. A. L. \& Melo, J. I. M. A família Leguminosae Juss. em dois afloramentos rochosos no município de Puxinanã, Paraíba. Biotemas, v. 26, n. 4, p. 23-43, 2013.

Silva, W. R. A importância das interações planta-animal nos processos de restauração. In: Kageyama, P. Y. et al. (org.). Restauração ecológica de ecossistemas naturais. Botucatu: Fundação de Estudos e Pesquisas Agrícolas e Florestais, 2003. p. 77-90.

Sobanski, N. \& Marques, C. M. M. Effects of soil characteristics and exotic grass cover on the forestrestoration of the Atlantic Forest. Journal for Nature Conservation, v. 1, p. 217-222, 2014. 
Society for Ecological Restoration. The SER International Primer on Ecological Restoration. Tucson: Society for Ecological Restoration International, International Science \& Policy Working Group, 2004. 15 p.

Souza, L. M. Regeneração natural como indicador da sustentabilidade em áreas em processo de restauração. 2014. Tese (Doutorado em Ciências Florestais) - Universidade Federal de Lavras, Lavras.

Suganuma, M. S. et al. Changes in plant species composition and functional traits along the successional trajectory of a restored patch of Atlantic Forest. Community Ecology, v. 15, n. 1, p. 27-36, 2014

Suganuma, M. S. et al. Ecossistemas de referência para restauração de matas ciliares: existem padrões de biodiversidade, estrutura florestal e atributos funcionais? Revista Árvore, v. 37, n. 5, p. 835-847, 2013.

Suganuma, M. S. et al. Environment and landscape rather than planting design are the drivers of sucess in long-term restoration of riparian Atlantic Forest. Applied Vegetation Science, v. 21, p. 76-84, 2018.
Talora, D. C. \& Morellato, L. P. C. Fenologia de espécies arbóreas em floresta de planície litorânea do sudeste do Brasil. Revista Brasileira de Botânica, v. 23, p. 13-26, 2000.

Urbanetz, C. et al. Floristic composition and similarity analysis of an Atlantic rain forest fragment in Cananéia, São Paulo state, Brazil. Revista Brasileira de Botânica, v. 33, p. 639-651, 2010.

Xavier, A. F. et al. Unidades de Conservação da Natureza no Estado de São Paulo. In: Rodrigues, R. R. \& Bononi, V. L. R. (org.). Diretrizes para a restauração da biodiversidade no Estado de São Paulo. São Paulo: Instituto de Botânica: Imprensa Oficial, 2008. p. 22-42

Ziparro, V. B. et al. Levantamento florístico de floresta Atlântica no sul do estado de São Paulo, Parque Estadual de Intervales, Base Saibadela. Biota neotropica, v. 5, n. 1, p. 1-24, 2005. 